\title{
Position Tracking OF UNDERACTUATED VEHICLES ${ }^{1}$
}

\author{
António Pedro Aguiar João Pedro Hespanha \\ Dept. Electrical \& Computer Engineering, Univ. of California \\ Santa Barbara, CA 93106-9560 \\ Email:\{aguiar, hespanha\}@ece.ucsb.edu
}

\begin{abstract}
This paper addresses the problem of position tracking for underactuated autonomous vehicles moving in either two or three-dimensional space. A nonlinear tracking controller is proposed for a general class of vehicles that yields global stability and exponential convergence of the position tracking error to a neighborhood of the origin that can be made arbitrarily small. The desired trajectory does not need to be of a particular type (e.g., trimming trajectories) and in fact can be any sufficiently smooth bounded curve parameterized by time. The control algorithm proposed builds upon Lyapunov techniques. To illustrate its potential, we describe two vehicle control applications: an hovercraft (moving on a planar surface) and an underwater vehicle (moving in threedimensional space). Simulation results are presented and discussed.
\end{abstract}

\section{Introduction}

Over the past two decades, there has been a considerable research effort in the area of trajectory tracking for nonlinear autonomous vehicles. Trajectory tracking problems are concerned with the design of control laws that force a vehicle to reach and follow a time parameterized reference (i.e., a geometric path with an associated timing law). The degree of difficulty involved in solving this problem is highly dependent on the configuration of the vehicle. For fully actuated systems, the trajectory tracking problem is now reasonably well understood $[17,13,22]$.

For underactuated vehicles, i.e., systems with a smaller number of control inputs than the number of independent generalized coordinates $[12,27]$, trajectory tracking is still an active research topic. The study of these systems is motivated by the fact that it is usually costly and often not even practical (due to weight, reliability, complexity, and efficiency considerations) to fully actuate autonomous vehicles. Typical examples of underactuated systems include robotic manipulators, wheeled robots, walking robots, spacecraft, aircraft, helicopters, missiles, surface vessels, and underwater vehicles. The tracking problem for underactuated vehicles is especially challenging because most of these systems are not fully feedback linearizable and exhibit nonholonomic constraints, therefore standard tools used to control nonlinear systems - such as feedback linearization and integrator backstepping - are not directly applicable. See [20] for a survey of these concepts and [6] for a framework

\footnotetext{
${ }^{1}$ This material is based upon work supported by the National Science Foundation under Grant No. ECS-0242798. The work of António Aguiar was supported by a Pos-Doc Fellowship PRAXIS XXI from the Portuguese Foundation of Science and Technology.
}

to study the controllability and the design of motion algorithms for underactuated Lagrangian systems on Lie groups.

The classical approach for trajectory tracking of underactuated vehicles utilizes local linearization and decoupling of the multi-variable model to steer the same number of degrees of freedom as the number of available control inputs, which can be done using standard linear (or nonlinear) control methods. Canudas de Wit et al. [7] and Fossen [9] contain surveys of various trajectory tracking control design methods for mobile robots and ocean vehicles, respectively. Alternative approaches include the linearization of the vehicle error dynamics about trajectories that lead to a timeinvariant linear system (also known as trimming trajectories) combined with gain scheduling and/or Linear Parameter Varying (LPV) design methodologies [24, 14, 25, 21]. The basic limitation of these approaches is that stability is only guaranteed in a neighborhood of the selected operating points. Moreover, performance can suffer significantly when the vehicle executes maneuvers that emphasize its nonlinearity and cross-couplings.

Nonlinear Lyapunov-based designs can overcome some of the limitations mentioned above. Several examples of nonlinear trajectory tracking controllers for marine underactuated vehicles have been reported in the literature $[11,18$, 26, 19, 3, 4]. Godhavn [11] proposed a backstepping-based continuous time-invariant state-feedback control law for an underactuated surface vessel. The control law provides exponential position tracking, as long as saturation of the inputs is avoided and the vehicle surge velocity is nonzero. However, Godhavn's approach requires the ship to move along straight lines or arcs of circles to guarantee stable zero dynamics for the nonlinear system. Toussaint et al. [26] extended this approach to design a trajectory tracking controller for a nonminimum phase underactuated surface vessel. As in [11], the desired trajectory is restricted to straight lines and circles and it is assumed that the ship is always in motion. Pettersen and Nijmeijer [18] consider the kinematics of a surface vehicle and design a time-varying control law for the surge and yaw inputs. Under this controller, the errors in position and orientation with respect to a reference trajectory of constant curvature is practically globally exponentially stabilized to zero. The control law in [18] does not require nonzero desired linear velocity.

Typically, tracking problems for autonomous vehicles are solved by designing control laws that make the vehicles track pre-specified feasible "state-space" trajectories, i.e., trajectories that specify the time evolution of linear and angular positions and velocities that are consistent with the vehicles' dynamics (e.g. [18, 15, 10, 19, 4]). This approach suffers from the drawback that usually the vehicles' 
dynamics exhibit complex nonlinear terms and significant uncertainty that make the task of computing a feasible trajectory hard. Fortunately, in practical applications one often only needs to track the desired position making it possible to bypass the computation of feasible state-space trajectories. This is the approach followed here. This type of approach was also considered by Alonge et al. [3] in the context of underactuated underwater vehicles, where conditions for asymptotic tracking are provided and an observer of the marine current is also proposed.

Motivated by the above considerations, this paper addresses the problem of position tracking of underactuated vehicles in both two and three-dimensional spaces. The main contribution is a design methodology to construct a nonlinear tracking controller that yields global stability and exponential convergence of the position tracking error to a neighborhood of the origin that can be made arbitrarily small. Furthermore, the desired trajectory does not need to be a trimming trajectory and can be any sufficiently smooth time-varying bounded curve, including the degenerate case of a constant trajectory (set-point). The control algorithm proposed builds on iterative Lyapunov-based techniques, in particular, integrator backstepping [16, 23]. The class of vehicles for which the design procedure is applicable is quite general and includes any vehicle modeled as a rigid-body moving in an ideal fluid, subject to a controlled force and two or three independent control torques.

Contrary to most of the approaches described above, the controller proposed does not suffer from geometric singularities due to the parameterization of its rotation matrix. This is possible because the attitude control problem is formulated directly in the group of rotations $S O(3)$. The literature on designing tracking control laws for underactuated vehicles directly in the configuration manifold (avoiding in this way geometric singularities) is relatively scarce. Noteworthy examples include [5, 10]. Bullo [5] describes a procedure to exponentially stabilize relative equilibria of mechanical systems. Relative equilibria for systems in three dimensional Euclidean space include straight lines, circles, and generic helices. Frazzoli et al. [10] propose a trajectory tracking control design for an autonomous helicopter using a backstepping algorithm.

\section{Problem Statement}

Consider an underactuated vehicle modeled as a rigid body subject to external forces and torques. Let $\{I\}$ be an inertial coordinate frame and $\{B\}$ a body-fixed coordinate frame whose origin is located at the center of mass of the vehicle. The configuration $(R, p)$ of the vehicle is an element of the Special Euclidean group $S E(3):=S O(3) \times \mathbb{R}^{3}$, where $R \in S O(3):=\left\{R \in \mathbb{R}^{3 \times 3}: R R^{\prime}=I_{3}, \operatorname{det}(R)=+1\right\}$ is a rotation matrix that describes the orientation of the vehicle by mapping body coordinates into inertial coordinates, and $p \in \mathbb{R}^{3}$ is the position of the origin of $\{B\}$ expressed in $\{I\}$. Let $S: \mathbb{R}^{3} \rightarrow s o(3):=\left\{M \in \mathbb{R}^{3 \times 3}: M^{\prime}=-M\right\}$ be the map that assigns to a vector $x=\left(x_{1}, x_{2}, x_{3}\right)^{\prime} \in \mathbb{R}^{3}$, the $3 \times 3$ skew-symmetric matrix $S(x)=\left[\begin{array}{ccc}0 & -x_{3} & x_{2} \\ x_{3} & 0 & -x_{1} \\ -x_{2} & x_{1} & 0\end{array}\right]$. Denoting the linear and angular velocities of the vehicle relative to $\{I\}$ expressed in $\{B\}$ by $v \in \mathbb{R}^{3}$ and $\omega \in \mathbb{R}^{3}$, respectively, the following kinematic relations apply

$$
\begin{aligned}
& \dot{p}=R v, \\
& \dot{R}=R S(\omega) .
\end{aligned}
$$

We consider here the class of underactuated vehicle models with dynamic equations of motion of the following form:

$$
\begin{aligned}
\mathbf{M} \dot{v} & =-S(\omega) \mathbf{M} v+f_{v}(v, \omega, R)+g_{1} u_{1}, \\
\mathbf{J} \dot{\omega} & =-S(v) \mathbf{M} v-S(\omega) \mathbf{J} \omega+f_{\omega}(v, \omega, R)+G_{2} u_{2},
\end{aligned}
$$

where $\mathbf{M} \in \mathbb{R}^{3 \times 3}$ and $\mathbf{J} \in \mathbb{R}^{3 \times 3}$ denote constant symmetric positive definite mass and inertia matrices; $u_{1} \in \mathbb{R}$ and $u_{2} \in$ $\mathbb{R}^{3}$ denote the control inputs, which act upon the system through a constant nonzero vector $g_{1} \in \mathbb{R}^{3}$ and a constant nonsingular matrix $G_{2} \in \mathbb{R}^{3 \times 3}$, respectively; and $f_{v}(v, \omega, R)$, $f_{\omega}(v, \omega, R)$ represents all the remaining forces and torques acting on the body. For the special case of an underwater vehicle, $\mathbf{M}$ and $\mathbf{J}$ also includes the so-called hydrodynamic added-mass $M_{A}$ and added-inertia $J_{A}$ matrices, respectively, i.e., $\mathbf{M}=M_{R B}+M_{A}, \mathbf{J}=J_{R B}+J_{A}$, where $M_{R B}$ and $J_{R B}$ are the rigid-body mass and inertia matrices, respectively.

The problem considered in this paper can be stated as follows:

Consider the class of underactuated vehicles represented by equations (1)-(2) and let $p_{d}:[0, \infty) \rightarrow \mathbb{R}^{3}$ be a given threetimes continuously differentiable bounded time-varying desired trajectory. Design a controller such that all the closedloop signals are globally bounded and the tracking error $\left\|p-p_{d}\right\|$ converges exponentially fast to a neighborhood of the origin that can be made arbitrarily small.

The following assumptions are assumed to hold:

Assumption 1 The function $f_{v}(v, \omega, R)$ is affine in $\omega$, i.e., $f_{v}(v, \omega, R)=f_{v_{1}}(v, R)+f_{v_{2}}(v, R) \omega, \quad \forall v, \omega \in \mathbb{R}^{3}, R \in S O(3)$.

Assumption 2 There exists a vector $\delta \in \mathbb{R}^{3}$ such that for every $t \geq 0, v \in \mathbb{R}^{3}, R \in S O(3)$, the following matrix is fullrank

$$
\mathcal{B}\left(R, v, \dot{p}_{d}, \delta\right):=\left[\begin{array}{ll}
g_{1} & S(\mathbf{M} \delta)+\Gamma\left(R, v, \dot{p}_{d}\right)
\end{array}\right] \in \mathbb{R}^{3 \times 4}
$$

where

$$
\Gamma\left(R, v, \dot{p}_{d}\right):=S\left(\mathbf{M} R^{\prime} \dot{p}_{d}\right)-\mathbf{M} S\left(R^{\prime} \dot{p}_{d}\right)+f_{v_{2}}(v, R) .
$$

These assumptions seem to hold for a large class of vehicle models, which includes the Hovercraft and the underwater vehicles discussed in Section 5.

\section{Controller Design}

This section proposes a nonlinear control law to solve the tracking problem. For the sake of clarity, control-Lyapunov functions are introduced recursively borrowing from the techniques of backstepping [16].

\section{Step 1. Coordinate transformation}

Consider the global diffeomorphic coordinate transformation

$$
e:=R^{\prime}\left(p-p_{d}\right),
$$

which expresses the tracking error $p-p_{d}$ in the body-fixed frame. The dynamic equation for the tracking error $e$ is given by $\dot{e}=-S(\omega) e+v-R^{\prime} \dot{p}_{d}$. 


\section{Step 2. Convergence of $e$}

We start by defining the control-Lyapunov function

$$
V_{1}:=\frac{1}{2} e^{\prime} e
$$

and computing its time derivative to obtain

$$
\dot{V}_{1}=e^{\prime}\left[v-R^{\prime} \dot{p}_{d}\right]
$$

We can regard $v$ as a virtual control that one would like to use to make $\dot{V}_{1}$ negative. This could be achieved, e.g., if we could set $v$ equal to $R^{\prime} \dot{p}_{d}-k_{e} \mathbf{M}^{-1} e$, for some positive constant $k_{e}$. To achieve this we introduce the error variable

$$
z_{1}:=v-R^{\prime} \dot{p}_{d}+k_{e} \mathbf{M}^{-1} e,
$$

that we would like to set to zero, and re-write (5) as $\dot{V}_{1}=$ $-k_{e} e^{\prime} \mathbf{M}^{-1} e+e^{\prime} z_{1}$.

Step 3. Backstepping for $z_{1}$

After straightforward algebraic manipulations, we conclude that the dynamic equation for the error $z_{1}$ can be written as

$$
\mathbf{M} \dot{z}_{1}=S\left(\mathbf{M} z_{1}\right) \omega+\Gamma\left(R, v, \dot{p}_{d}\right) \omega+g_{1} u_{1}+h\left(e, R, v, z_{1}, \ddot{p}_{d}\right),
$$

where $\Gamma$ is defined in (4) and

$$
h\left(e, R, v, z_{1}, \ddot{p}_{d}\right):=f_{v_{1}}(v, R)-\mathbf{M} R^{\prime} \ddot{p}_{d}+k_{e} z_{1}-k_{e}^{2} \mathbf{M}^{-1} e .
$$

It turns out that it will not always be possible to drive $z_{1}$ to zero. Instead, we will drive $z_{1}$ to the constant $\delta$ in Assumption 2. To achieve this we define $\varphi:=z_{1}-\delta$ as a new error variable that we will drive to zero and consider the augmented control-Lyapunov function

$$
V_{2}:=\frac{1}{2} e^{\prime} e+\frac{1}{2} \varphi^{\prime} \mathbf{M}^{2} \varphi=V_{1}+\frac{1}{2} \varphi^{\prime} \mathbf{M}^{2} \varphi
$$

The time derivative of $V_{2}$ can be written as

$$
\begin{aligned}
\dot{V}_{2}= & -k_{e} e^{\prime} \mathbf{M}^{-1} e+e^{\prime} \delta+\varphi^{\prime}\left(\mathbf{M} \mathcal{B}\left(R, v, \dot{p}_{d}, \delta\right) \mu\right. \\
& \left.+\mathbf{M} h\left(e, R, v, z_{1}, \ddot{p}_{d}\right)+e\right),
\end{aligned}
$$

where $\mathcal{B}$ is defined in (3) and $\mu:=\left[\begin{array}{ll}u_{1} & \omega^{\prime}\end{array}\right]^{\prime} \in \mathbb{R}^{4}$. One can now regard $\mu$ as a virtual control (actually its first component is already a "real" control) that one would like to use to make $\dot{V}_{2}$ negative. This could be achieved, e.g., if one could set $\mu$ equal to ${ }^{1}$

$$
\alpha:=\mathcal{B}^{\prime}\left(\mathcal{B B}^{\prime}\right)^{-1}\left(-h\left(e, R, v, z_{1}, \ddot{p}_{d}\right)-\mathbf{M}^{-1} e-\mathbf{M}^{-1} K_{\varphi} \varphi\right),
$$

where $K_{\varphi} \in \mathbb{R}^{3 \times 3}$ is a symmetric positive definite matrix. To achieve this we set $u_{1}$ to be equal to the first entry of $\alpha$, i.e.,

$$
u_{1}=\left[\begin{array}{ll}
1 & 0_{1 \times 3}
\end{array}\right] \alpha
$$

introduce the error variable $z_{2}:=\omega-\left[0_{3 \times 1} I_{3 \times 3}\right] \alpha$ that one would like to set to zero, and re-write (7), with $u_{1}$ given by (8), as

$$
\dot{V}_{2}=-k_{e} e^{\prime} \mathbf{M}^{-1} e+e^{\prime} \delta-\varphi^{\prime} K_{\varphi} \varphi+\varphi^{\prime} \mathbf{M} \mathcal{B}_{b}\left(R, v, \dot{p}_{d}, \delta\right) z_{2}
$$

\footnotetext{
${ }^{1}$ We recall that $\mathcal{B B}^{\prime}$ is nonsingular because of Assumption 2.
}

where $\mathcal{B}_{b} \in \mathbb{R}^{3 \times 3}$ denotes the sub-matrix formed by the last three columns of $\mathcal{B}$, i.e.,

$$
\mathcal{B}_{b}\left(R, v, \dot{p}_{d}, \delta\right):=S(\mathbf{M} \delta)+\Gamma\left(R, v, \dot{p}_{d}\right) \in \mathbb{R}^{3 \times 4}
$$

\section{Step 4. Backstepping for $z_{2}$}

Consider now a third control-Lyapunov function given by

$$
V_{3}:=\frac{1}{2} e^{\prime} e+\frac{1}{2} \varphi^{\prime} \mathbf{M}^{2} \varphi+\frac{1}{2} z_{2}^{\prime} \mathbf{J} z_{2}=V_{2}+\frac{1}{2} z_{2}^{\prime} \mathbf{J} z_{2} .
$$

Computing its time derivative one obtains

$$
\begin{aligned}
\dot{V}_{3}= & -k_{e} e^{\prime} \mathbf{M}^{-1} e+e^{\prime} \delta-\varphi^{\prime} K_{\varphi} \varphi+ \\
& +z_{2}^{\prime}\left(G_{2} u_{2}-S(v) \mathbf{M} v-S(\omega) \mathbf{J} \omega+f_{\omega}(v, \omega, R)\right. \\
& \left.-\left[0_{3 \times 1} \mathbf{J}\right] \dot{\alpha}+\mathcal{B}_{b}\left(R, v, \dot{p}_{d}, \delta\right)^{\prime} \mathbf{M} \varphi\right) .
\end{aligned}
$$

For simplicity we did not expand the derivative of $\alpha$. If we then choose

$$
\begin{aligned}
u_{2}= & G_{2}^{-1}\left(S(v) \mathbf{M} v+S(\omega) \mathbf{J} \omega-f_{\omega}(v, \omega, R)\right. \\
& \left.+\left[0_{3 \times 1} \mathbf{J}\right] \dot{\alpha}-\mathcal{B}_{b}\left(R, v, \dot{p}_{d}, \delta\right)^{\prime} \mathbf{M} \varphi-K_{z_{2}} z_{2}\right),
\end{aligned}
$$

the time derivative of $V_{3}$ becomes

$$
\dot{V}_{3}=-k_{e} e^{\prime} \mathbf{M}^{-1} e+e^{\prime} \delta-\varphi^{\prime} K_{\varphi} \varphi-z_{2}^{\prime} K_{z_{2}} z_{2} .
$$

Note that although $\dot{V}_{3}$ is not necessarily always negative, this will be sufficient to achieve practical stability.

\section{Stability analysis}

In this section, we prove that the control signals defined above are well defined, bounded, and that the tracking error converges exponential to an arbitrarily small neighborhood of the origin. The following is the key result of this paper.

Theorem 1 Given a three-times continuously differentiable bounded time-varying desired trajectory $p_{d}:[0, \infty) \rightarrow \mathbb{R}^{3}$, consider the closed-loop system $\Sigma$ consisting of the underactuated vehicle model (1)-(2) and the feedback controller (8), (10).

i) For any initial condition the solution to $\Sigma$ exists globally, all closed-loop signals are bounded, and the tracking error $\left\|p(t)-p_{d}(t)\right\|$ satisfies

$$
\left\|p(t)-p_{d}(t)\right\| \leq e^{-\lambda t} c_{0}+\varepsilon,
$$

where $\lambda, c_{0}, \varepsilon$ are positive constants. From these, only $c_{0}$ depends on initial conditions.

ii) By appropriate choice of the controller parameters $k_{e}, K_{\varphi}, K_{z_{2}}$, any desired values for $\varepsilon$ and $\lambda$ in (12) are possible.

Proof: To prove (i) we use Young's inequality to conclude that for any $\gamma>0$,

$$
\dot{V}_{3} \leq-e^{\prime}\left(k_{e} \mathbf{M}^{-1}-\frac{\gamma}{2} I\right) e-\varphi^{\prime} K_{\varphi} \varphi-z_{2}^{\prime} K_{z_{2}} z_{2}+\frac{1}{2 \gamma}\|\delta\|^{2} .
$$


Suppose now that we choose $\gamma$ sufficiently small so that the matrix $k_{e} \mathbf{M}^{-1}-\frac{\gamma}{2} I$ is positive definite. In this case, we conclude that there is a sufficiently small positive constant $\lambda$ such that

$$
\dot{V}_{3} \leq-\lambda V_{3}+\frac{1}{2 \gamma}\|\delta\|^{2}
$$

and therefore it is straightforward to conclude from the Comparison Lemma that

$$
V_{3}(t) \leq e^{-\lambda t} V_{3}(0)+\frac{1}{2 \lambda \gamma}\|\delta\|^{2}
$$

along solutions to $\Sigma$. From here we conclude that all signals remain bounded and therefore the solution exists globally. Moreover, $V_{3}$ converges to $\frac{1}{2 \lambda \gamma}\|\delta\|^{2}$ and therefore $\|e\|$ converges to a ball of radius $\frac{\|\delta\|}{\sqrt{\lambda \gamma}}$, because of (9).

To prove (ii), we show next that it is actually possible to make the radius $\frac{\|\delta\|}{\sqrt{\lambda \gamma}}$ as small as we want by appropriately choosing the controller parameters. To this effect, suppose we pick a desired radius $\varepsilon$ and a convergence rate $\lambda>0$. We can then define $\gamma:=\frac{\|\delta\|^{2}}{\varepsilon^{2} \lambda}$, provided that we choose $k_{e}$ sufficiently large so that $k_{e} \mathbf{M}^{-1}-\frac{\gamma}{2} I=k_{e} \mathbf{M}^{-1}-\frac{\|\delta\|^{2}}{2 \varepsilon^{2} \lambda} I \geq \frac{\lambda}{2} I>0$. If we then select $K_{\varphi}:=\frac{\lambda}{2} \mathbf{M}^{2}, K_{z_{2}}:=\frac{\lambda}{2} \mathbf{J}$, we conclude from (13) that (14) indeed holds for the pre-specified $\lambda$, from which (15) follows. However, now the above choices for the parameters lead to the desired radius $\frac{\|\delta\|}{\sqrt{\lambda \gamma}}=\varepsilon$.

Remark 1 We did not impose any constraints on the desired trajectory (besides being sufficiently smooth and its derivative being bounded) and we also did not require that the linear velocity of the vehicle to be always non-null. Consequently, $p_{d}(t)$ can be an arbitrary trajectory (which does not need to satisfy the dynamic model of the vehicle) and in particular can be constant for all $t \geq t_{0}$. In that case, the controller solves the position regulation problem.

\section{Illustrative examples}

This section illustrates the designs techniques and the stability results presented so far. We describe two vehicle control applications: an hovercraft (moving on a planar surface) and an underwater vehicle (moving in three-dimensional space). Simulation results are presented and discussed.

\subsection{Example 1. Position tracking of an underactuated Hovercraft}

Consider the vehicle described in [8] consisting of a platform mounted on three low-friction, omni-directional casters, over which are attached two high-performance ducted fans. Let $p=(x, y)^{\prime} \in \mathbb{R}^{2}$ be the Cartesian coordinate of the vehicle's center of mass and $\theta \in \mathbb{S}^{1}$ its orientation. Assuming that the friction forces and moments can be modeled by viscous friction, the equations of motion are [8]:

$$
\begin{aligned}
m \ddot{x} & =-d_{v} \dot{x}+\left(F_{s}+F_{p}\right) \cos \theta \\
m \ddot{y} & =-d_{v} \dot{y}+\left(F_{s}+F_{p}\right) \sin \theta \\
J \ddot{\theta} & =-d_{\omega} \dot{\theta}+l\left(F_{s}-F_{p}\right)
\end{aligned}
$$

where $m=5.5 \mathrm{~kg}$ is the mass of the vehicle and $J=$ $0.047 \mathrm{Kg} \mathrm{m}^{2}$ is the rotational inertia. The starboard and portboard fan forces are denoted $F_{s}$ and $F_{p}$, respectively, and $l=0.123 m$ denotes the moment arm of the forces where the geometry and mass centers of the vehicle are assumed to coincide. See [8] for details. The coefficient of viscous friction, $d_{v}$, is 4.5 and the coefficient of rotational friction, $d_{\omega}$, is 0.41 .

Expressing the equations of motion in the body fixed frame, yields

$$
\begin{aligned}
\dot{p} & =R(\theta) v \\
\dot{\theta} & =\omega \\
M \dot{v} & =-S(\omega) M v-D_{v} v+g_{1} u_{1} \\
J \dot{\omega} & =-d_{\omega} \omega+u_{2}
\end{aligned}
$$

where $v=(u, v)^{\prime}$ denotes the body-fixed linear velocities in surge and sway, $S(\omega)=\left(\begin{array}{cc}0 & -\omega \\ \omega & 0\end{array}\right), R(\theta)=\left(\begin{array}{cc}\cos \theta & -\sin \theta \\ \sin \theta & \cos \theta\end{array}\right), M=$ $\operatorname{diag}\{m, m\}, D_{v}=\operatorname{diag}\left\{d_{v}, d_{v}\right\}, g_{1}=(1,0)^{\prime}, u_{1}=F_{s}+F_{p}$, and $u_{2}=l\left(F_{s}-F_{p}\right)$.

Since $M=m I$, it follows that $\Gamma(\cdot)$ given by equation (4) is zero. Notice also that $D_{v}>0$. This will be exploited shortly. Following the general procedure presented in Section 3, the time derivative of $V_{2}$ is given by

$$
\dot{V}_{2}=-\frac{k_{e}}{m}\|e\|^{2}+e^{\prime} \delta-\varphi^{\prime} M D_{v} \varphi+\varphi^{\prime}[M \mathcal{B} \mu+e+M h(\cdot)]
$$

where $\delta=\left(\delta_{1}, \delta_{2}\right)^{\prime} \in \mathbb{R}^{2}, \varphi=z_{1}-\delta, z_{1}=v-R^{\prime}(\theta) \dot{p}_{d}+\frac{k_{e}}{m} e$, $\mathcal{B}=\left(\begin{array}{cc}1 & -m \delta_{2} \\ 0 & m \delta_{1}\end{array}\right), \mu=\left(u_{1}, \omega\right)^{\prime}$, and $h(\cdot)=-D_{v}\left[\delta+R^{\prime} \dot{p}_{d}-\right.$ $\left.\frac{k_{e}}{m} e\right]-M R^{\prime} \ddot{p}_{d}+k_{e} z_{1}-\frac{k_{e}^{2}}{m} e$. Clearly, the matrix $\mathcal{B}$ is nonsingular for any $\delta_{1}$ non zero. Therefore, choosing $\alpha=$ $-\mathcal{B}^{-1}\left[M^{-1} e+h(\cdot)+M^{-1} K_{\varphi} \varphi\right], \quad u_{1}=[1,0] \alpha, z_{2}=\omega-$ $[0,1] \alpha, u_{2}=-\mathcal{B}_{b}^{\prime} M \varphi+d_{\omega} \alpha_{2}+J \dot{\alpha}_{2}-k_{z_{2}} z_{2}$, where the $2 \times 2$ symmetric matrix $K_{\varphi}$ is positive definite, and $\mathcal{B}_{b}$ denotes the second column of $\mathcal{B}$, we get

$$
\dot{V}_{3}=-\frac{k_{e}}{m}\|e\|^{2}+e^{\prime} \delta-\varphi^{\prime} D_{v} M \varphi-\varphi^{\prime} K_{\varphi} \varphi-d_{\omega} z_{2}^{2}-k_{z_{2}} z_{2}^{2}
$$

which suffices to achieve practical stability.

Simulation results: We now illustrate the performance of the proposed control scheme through computer simulation. The objective is to force the hovercraft to track the "virtual" kinematic unicycle vehicle

$$
\dot{x}_{d}=u_{d} \cos \theta_{d}, \quad \dot{y}_{d}=u_{d} \sin \theta_{d}, \quad \dot{\theta}_{d}=\omega_{d},
$$

which starts with the pose $x_{d}\left(t_{0}\right)=y_{d}\left(t_{0}\right)=\theta_{d}\left(t_{0}\right)=0$ and moves with velocities $u_{d}(t)=0.2 \mathrm{~m} / \mathrm{s}$ and $\omega_{d}(t)=$ $0.1 \mathrm{rad} / \mathrm{s}$. The initial conditions for the hovercraft are $(x, y, \theta, u, v, \omega)=(-0.2 m,-1 m, 0,0,0,0)$. The control parameters were selected as follows: $k_{e}=0.4, K_{\varphi}=2 I$, $k_{z_{2}}=2$, and $\delta=(-0.05,0)^{\prime}$. Figure 1 depicts the reference and hovercraft trajectories in the $x y$-plane. As expected, the hovercraft converges to a neighborhood of the "virtual" unicycle vehicle. Figure 2 shows the time evolution of some relevant variables. Notice that the desired trajectory $p_{d}$ can be any arbitrary sufficiently smooth bounded trajectory and that we are not imposing any other constraints such as, e.g., that the desired trajectory must satisfy the dynamic model 
of the vehicle. Consequently, the vehicle generally will not be aligned (in steady-state) with the direction of the tangent velocity of $p_{d}$ as it can be seen in Figure 1 together with Figure 2. Therefore, contrary to what happen in the field of wheeled mobile robots (where it is assumed that the lateral drag coefficient $d_{v}=+\infty$ ) we can not force $\theta$ to converge to the direction of the tangent velocity $p_{d}$.

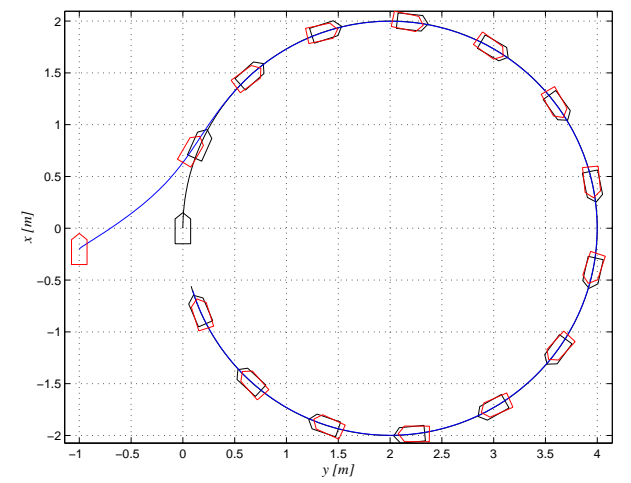

Figure 1: Trajectories of the hovercraft and reference for a "virtual" unicycle vehicle in the $x y$-plane. Initial conditions for the hovercraft: $(x, y, \theta, u, v, \omega)=$ $(-0.2 m,-1 m, 0,0,0,0)$; and initial pose for the "virtual" unicycle vehicle: $x_{d}=y_{d}=\theta_{d}=0$.

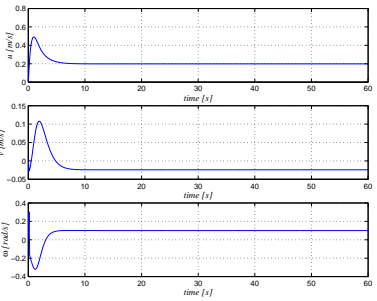

(a)

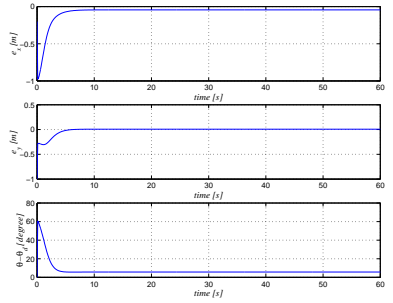

(b)
Figure 2: Time evolution of (a) linear velocity in $x$-direction, in $y$-direction, and angular velocity $\omega(t)$; and (b) tracking error in $x$-direction, in $y$-direction, and $\tilde{\theta}=\theta-\theta_{d}$.

\subsection{Example 2. Position tracking of an underwater ve- hicle in 3-D space}

Consider an ellipsoid shape underactuated autonomous underwater vehicle (AUV) not necessarily neutrally buoyant. Let $\{B\}$ be a body-fixed coordinate frame whose origin is located at the center of mass of the vehicle. Suppose that we have available a pure body-fixed control force $\tau_{u}$ in the $x_{B}$ direction, and two independent control torques $\tau_{q}$ and $\tau_{r}$ about the $y_{B}$ and $z_{B}$ axes of the vehicle, respectively. The following notation is used [9]:

$p=[x, y, z]^{\prime}-$ position of the origin of $\{B\}$ measured in $\{I\}$.

$R \in S O(3)$ - rotation matrix that describes the orientation of $\{B\}$ with respect to $\{I\}$.

$v_{1}=[u, v, w]^{\prime}$ - linear velocity of the origin of $\{B\}$ relative to $\{I\}$, expressed in $\{B\}$ (i.e., body-fixed linear velocity).

$v_{2}=[p, q, r]^{\prime}-$ angular velocity of $\{B\}$ relative to $\{I\}$, expressed in $\{B\}$ (i.e., body-fixed angular velocity).

$r_{B}=\left[r_{x_{B}}, r_{y_{B}}, r_{z_{B}}\right]^{\prime}-$ position of the center of buoyancy measured in $\{I\}$.
The kinematics and dynamics equations of motion of the vehicle can be written as

$$
\begin{aligned}
\dot{p} & =R v_{1}, \\
\dot{R} & =R S\left(v_{2}\right), \\
\mathbf{M} \dot{v}_{1} & =-S\left(v_{2}\right) \mathbf{M} v_{1}-D_{v_{1}}\left(v_{1}\right) v_{1}-g_{1}(R)+B_{1} u_{1}, \\
\mathbf{J} \dot{v}_{2} & =-S\left(v_{1}\right) \mathbf{M} v_{1}-S\left(v_{2}\right) \mathbf{J} v_{2}-D_{v_{2}}\left(v_{2}\right) v_{2}-g_{2}(R)+B_{2} u_{2},
\end{aligned}
$$

where $\mathbf{M}=\operatorname{diag}\left\{m_{11}, m_{22}, m_{33}\right\}, \mathbf{J}=\operatorname{diag}\left\{J_{11}, J_{22}, J_{33}\right\}$, $u_{1}=\tau_{u}, u_{2}=\left(\tau_{q}, \tau_{r}\right)^{\prime}, D_{v_{1}}\left(v_{1}\right)=\operatorname{diag}\left\{X_{u}+X_{|u| u}|u|, Y_{v}+\right.$ $\left.Y_{|v| v}|v|, Z_{w}+Z_{|w| w}|w|\right\}, D_{v_{2}}\left(v_{2}\right)=\operatorname{diag}\left\{K_{p}+K_{|p| p}|p|, M_{q}+\right.$ $\left.M_{|q| q}|q|, N_{r}+N_{|r| r}|r|\right\}, B_{1}=\left(\begin{array}{l}1 \\ 0 \\ 0\end{array}\right), B_{2}=\left(\begin{array}{ll}0 & 0 \\ 1 & 0 \\ 0 & 1\end{array}\right), g_{1}(R)=$ $R^{\prime}\left(\begin{array}{c}0 \\ 0 \\ W-B\end{array}\right), g_{2}(R)=S\left(r_{B}\right) R^{\prime}\left(\begin{array}{l}0 \\ 0 \\ B\end{array}\right)$. The gravitational and buoyant forces are given by $W=m g$ and $B=\rho g \nabla$, respectively, where $m$ is the constant mass, $\rho$ is the mass density of the water and $\nabla$ is the volume of the displaced water.

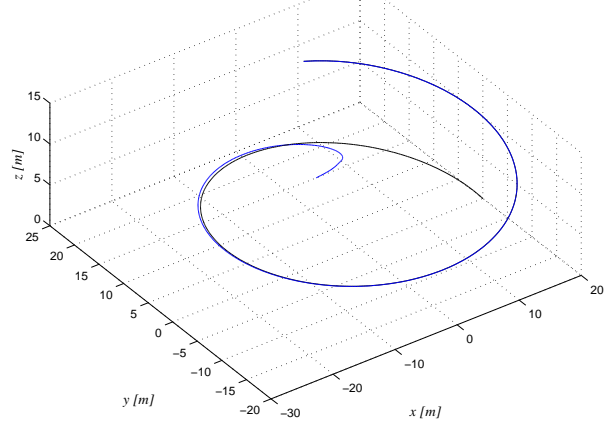

Figure 3: Vehicle trajectory in 3-D space. The desired trajectory is a helix and the initial conditions of the AUV are $(x, y, z)=(15 m, 5 m, 0), R=I$, and $v_{1}=v_{2}=0$.

In this example the time derivative of $V_{2}$ yields

$$
\begin{aligned}
\dot{V}_{2}= & -k_{e} e^{\prime} M^{-1} e+e^{\prime} \delta-\varphi^{\prime} D_{v_{1}} \mathbf{M}\left(v_{1}\right) \varphi \\
& +\varphi^{\prime}\left[\mathbf{M} \mathcal{B} \mu+\mathbf{M}\left(\Gamma_{a}-S_{a}(\delta)\right) p+e+\mathbf{M} h(\cdot)\right],
\end{aligned}
$$

where $\Gamma_{a}$ and $S_{a}(\delta)$ denote the first columns of the matrices $\Gamma$ and $S(\delta)$, respectively, $\mu=\left[u_{1}, q, r\right]^{\prime}, \delta=\left(\delta_{1}, \delta_{2}, \delta_{3}\right)^{\prime} \in$ $\mathbb{R}^{3}$, and $h(\cdot)=-D_{v_{1}}\left(v_{1}\right)\left[\delta+R^{\prime} \dot{p}_{d}-k_{e} \mathbf{M}^{-1} e\right]-g_{1}(R)-$ $\mathbf{M} R^{\prime} \ddot{p}_{d}+k_{e} z_{1}-k_{e}^{2} \mathbf{M}^{-1} e$. The terms associated with the angular velocity $p$ were moved out of the matrix $\mathcal{B}$ since the vehicle is not directly actuated in roll. Thus,

$$
\mathcal{B}=\left(\begin{array}{ccc}
1 & -\left(m_{33}-m_{11}\right) a_{3}+\delta_{3} & \left(m_{22}-m_{11}\right) a_{2}-\delta_{2} \\
0 & 0 & -\left(m_{11}-m_{22}\right) a_{1}+\delta_{1} \\
0 & \left(m_{11}-m_{33}\right) a_{1}-\delta_{1} & 0
\end{array}\right),
$$

where $a=\left(a_{1}, a_{2}, a_{3}\right)^{\prime}:=R^{\prime} \dot{p}_{d}$. It is straightforward to show that $\mathcal{B}$ is nonsingular for any $\delta_{1} \geq\left|a_{1}\right| \max \left(\mid m_{11}-\right.$ $\left.m_{22}|,| m_{11}-m_{33} \mid\right)$. Notice that $\left|a_{1}\right| \leq\left\|\dot{p}_{d}\right\|$, which it is assumed to be bounded.

Simulation results: To illustrate the performance of the tracking control algorithm, computer simulations were carried out with a model of the Sirene AUV assuming that the vehicle is directly actuated in force in the $x_{B}$ direction (control variable $\tau_{u}$ ), and in torque about the $y_{B}$ and $z_{B}$ axes, (controls $\tau_{q}$ and $\tau_{r}$ ), respectively. The vehicle dynamic model can be found in [2]. See also [1], for complete details.

Figure 3 displays the resulting vehicle trajectory in the $3 \mathrm{D}$ space for the helix desired trajectory $p_{d}(t)=\left[v_{1} \cos \left(\frac{2 \pi}{T}(t-\right.\right.$ 
$\left.\left.\left.t_{0}\right)\right), v_{1} \sin \left(\frac{2 \pi}{T}\left(t-t_{0}\right)\right), v_{2}\left(t-t_{0}\right)\right]^{\prime}$, with $v_{1}=20, v_{2}=0.1$ and $T=100$. The initial conditions of the AUV are $(x, y, z)=$ $(15 m, 5 m, 0), R=I$, and $v_{1}=v_{2}=0$. The control parameters were selected as follows: $k_{e}=m 0.1, K_{\varphi}=I, K_{z_{2}}=I$, $\varepsilon=0.5$, and $\delta=\left(-2 \max \left(\left|m_{11}-m_{22}\right|,\left|m_{11}-m_{33}\right|\right), 0,0\right)^{\prime}$. Figure 4 shows the time evolution of the tracking error $e$, the Euler angles (computed from $R \in S O(3)$ ), and the time evolution of the linear and angular velocities. The damped oscillatory behavior of pitch and roll are due to the gravitational and buoyancy forces.

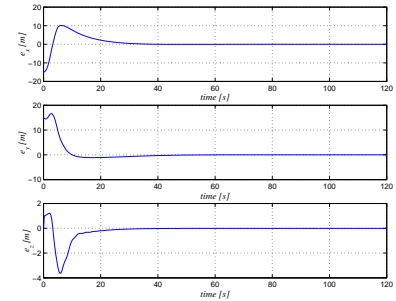

(a)

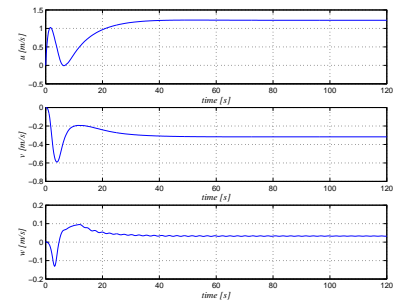

(c)

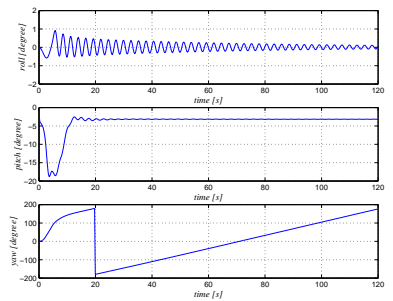

(b)

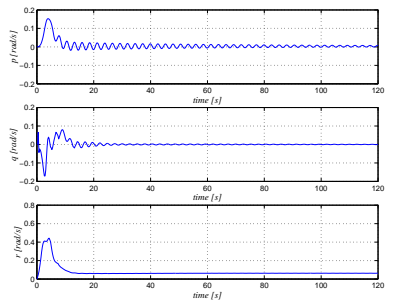

(d)
Figure 4: Time evolution of (a) tracking error $e=\left(e_{x}, e_{y}, e_{z}\right)^{\prime}$; (b) roll $\phi$, pitch $\theta$, yaw $\psi$ Euler angles; (c) body-fixed linear velocities $u(t), v(t)$, and $w(t)$; and (d) body-fixed angular velocities $p(t), q(t)$, and $r(t)$.

\section{Conclusions}

We proposed a solution to the position tracking problem for underactuated autonomous vehicles moving in either two or three-dimensional spaces. The control algorithm derived builds upon Lyapunov techniques and it was shown to yield global stability and exponential convergence of the position tracking error to a neighborhood of the origin that can be made arbitrarily small. The desired trajectory does not need to be of a particular type (e.g., trimming trajectories) and in fact can be any sufficiently smooth bounded curve parameterized by time. To illustrate the results presented, we describe two vehicle control applications: an hovercraft (moving on a planar surface) and an underwater vehicle (moving in three-dimensional space). Simulation results show that the control objectives are accomplished.

Future research will address the extension of these results to a larger class of models by relaxing the requirements imposed by Assumptions 1 and 2. Another open problem that warrants further research is the control of underactuated vehicles with noise and model uncertainties that arise from parameter variations or neglected dynamics.

\section{References}

[1] A. P. Aguiar. Nonlinear Motion Control of Nonholonomic and Underactuated Systems. PhD thesis, Dept. Electrical Engineering, Instituto Superior Técnico, IST, Lisbon, Portugal, 2002.

[2] A. P. Aguiar and A. M. Pascoal. Modeling and control of an autonomous underwater shuttle for the transport of benthic laborato- ries. In Proc. of the Oceans 97 Conference, Halifax, Nova Scotia, Canada, Oct. 1997.

[3] F. Alonge, F. D'Ippolito, and F. Raimondi. Trajectory tracking of underactuated underwater vehicles. In Proc. 40th IEEE Conference on Decision and Control, Orlando, Florida, USA, Dec. 2001.

[4] A. Behal, D. Dawson, W. Dixon, and Y. Fang. Tracking and regulation control of an underactuated surface vessel with nonintegrable dynamics. IEEE Trans. on Aut. Control, 47(3):495-500, Mar. 2002.

[5] F. Bullo. Stabilization of relative equilibria for underactuated systems on riemannian manifolds. Automatica, 36:1819-1834, 2000.

[6] F. Bullo, N. E. Leonard, and A. D. Lewis. Controllability and motion algorithms for underactuated lagrangian systems on lie groups. IEEE Transactions on Automatic Control, 45(8):1437-1454, 2000.

[7] C. Canudas de Wit, B. Siciliano, and G. Bastin, Eds. Theory of robot control. Communications and control eng. Springer-Verlag, 1997.

[8] L. Cremean, W. Dumbar, D. van Gogh, J. Hickey, E. Klavins, J. Meltzer, and R. Murray. The caltech multi-vehicle wireless testbed. In Proc. 41st IEEE Conf. on Decision and Control, Las Vegas, NV, Dec. 2002.

[9] T. I. Fossen. Guidance and Control of Ocean Vehicles. John Wiley \& Sons, England, 1994.

[10] E. Frazzoli, M. Dahleh, and E. Feron. Trajectory tracking control design for autonomous helicopters using a backstepping algorithm. In Proc. of the Amer. Control Conference, Chicago, IL, June 2000.

[11] J. M. Godhavn. Nonlinear tracking of underactuated surface vessels. In Proc. 35th IEEE Conference on Decision and Control, pages 975-980, Kobe, Japan, Dec. 1996.

[12] H. Goldstein. Classical Mechanics. Addison-Wesley, Reading, MA, USA, $2^{\text {nd }}$ edition, 1980. 672 pages.

[13] A. Isidori. Nonlinear Control Systems. Springer-Verlag, London, UK, $3^{\text {rd }}$ edition, 1989.

[14] I. Kaminer, A. Pascoal, E. Hallberg, and C. Silvestre. Trajectory tracking controllers for autonomous vehicles: An integrated approach to guidance and control. Journal of Guidance, Control, and Dynamics, 21(1):29-38, 1998.

[15] T. Koo and S. Sastry. Output tracking control design of a helicopter model based on approximate linearization. In Proc. 37th IEEE Conf. on Decision and Control, pages 3635-3640, Tampa, FL, Dec. 1998.

[16] M. Krstić, I. Kanellakopoulos, and P. Kokotović. Nonlinear and Adaptive Control Design. John Wiley \& Sons, Inc., 1995.

[17] H. Nijmeijer and A. J. van der Schaft. Nonlinear Dynamical Control Systems. Springer-Verlag, New York, USA, 1990.

[18] K. Y. Pettersen and H. Nijmeijer. Global practical stabilization and tracking for an underactuated ship - a combined averaging and backstepping approach. In Proc. IFAC Conference on Systems Structure and Control, pages 59-64, Nantes, France, July 1998.

[19] K. Y. Pettersen and H. Nijmeijer. Underactuated ship tracking control: theory and experiments. International Journal of Control, 74 (14):1435-1446, 2001.

[20] M. Reyhanoglu, A. van der Schaft, N. H. McClamroch, and I. Kolmanovsky. Dynamics and control of a class of underactuated mechanical systems. IEEE Tr. on Aut. Cont., 44(9):1663-1671, 1999.

[21] W. J. Rugh and J. S. Shamma. Research on gain-scheduling. Automatica, 36(10):1401-1425, 2000.

[22] S. Sastry. Nonlinear Systems: Analysis, Stability, and Control. Interdisciplinary Applied Mathematics: Systems and Control. Springer, New York, 1999.

[23] R. Sepulchre, M. Janković, and P. Kokotović. Constructive Nonlinear Control. Springer-Verlag, New York, USA, 1997.

[24] J. S. Shamma and J. R. Cloutier. Gain-scheduled missile autopilot design using linear parameter varying transformations. Journal of Guidance, Control, and Dynamics, 16(2):256-263, 1993.

[25] C. Silvestre. Multi-objective optimization theory with applications to the integrated design of controllers/plants for autonomous vehicles. PhD thesis, Inst. Superior Técnico, Lisbon, Portugal, 2000.

[26] G. Toussaint, T. Basar, and F. Bullo. Tracking for nonlinear underactuated surface vessels with generalized forces. In Proc. of the 2000 IEEE International Conference on Control Applications, pages 355-360, Anchorage, AK, USA, Sept. 2000.

[27] J. Wen. Control of nonholonomic systems. In The Control Handbook, pages 1359-1368, 1996. CRC Press \& IEEE Press. 